\title{
Kinder und Jugendliche mit Behinderungen in der Kommune - Kommentar aus Sicht einer Schule
}

\author{
Martina Weide-Gertke und Brigitta Seidenschwang
}

Das Ziel einer inklusiven Schule, dem sich auch die Ernst-Barlach-Schulen der Stiftung Pfennigparade verpflichtet haben, muss es sein, den Schülerinnen und Schülern größtmögliche Selbstständigkeit für ihre körperliche, geistige und soziale Entwicklung zu eröffnen. Es geht darum, sie auf eine möglichst realistische Einschätzung ihrer eigenen Möglichkeiten und Fähigkeiten, eine qualifizierte Berufswahl, den Übertritt in weitere schulische Bildungsgänge bis zur Hochschulreife, eine Teilhabe am gesellschaftlichen Leben und eine eigenständige Lebensführung vorzubereiten.

Hinzukommen muss außerdem die Integration Nichtbehinderter, um behinderten Schülern ein möglichst alltagsnahes Umfeld zu schaffen bzw. ihnen den Umgang mit Nichtbehinderten zu ermöglichen.

Nichtbehinderte Jugendliche bringen andere Vorerfahrungen mit, die im schulischen Zusammenleben für behinderte Jugendliche entwicklungsfördernd sind. Besonders im Bereich der Selbstwahrnehmung/Einschätzung ist das Feedback durch Gleichaltrige sehr wichtig. Zudem ist die Hilfe durch Mitschüler in der Regel sozial fördernder als die Hilfe durch professionelle Erwachsene. Zu lernen ist vor allem, angemessen Hilfen einzufordern, das Nehmen und Geben und sich um weitestgehende Selbstständigkeit zu bemühen. Behinderte und nichtbehinderte Jugendliche können über das schulische $\mathrm{Zu}$ sammenleben hinaus dauerhafte Beziehungen aufbauen.

Wir erleben, dass die Begegnung zwischen behinderten und nichtbehinderten Menschen ein großes Wachstumspotential für die Entwicklung ihrer Persönlichkeit enthält.

Die Grundlagen unseres pädagogischen Handelns sind die Wahrnehmung jedes Einzelnen und der Respekt vor der Besonderheit der uns anvertrauten jungen Menschen. Wir unterstützen und fördern die Stärken unserer Schüler, denn Lernen und Entwicklung brauchen Ermutigung.

Wir schaffen einerseits Lernbedingungen, welche die Eigenaktivität und Selbstbestimmung stärken. Dazu bringen wir junge Menschen in herausfordernde Situationen, die sie auch bestehen können. Andererseits setzen wir uns mit Abhängigkeiten und Begrenztheiten auseinander.

Für den Unterricht bedeutet dies ganz konkret, flexibel mit inhaltlichen und zeitlichen Konzepten umzugehen, „Störungen" mit Geduld zu begegnen, kleinste Entwicklungsfortschritte genau zu beobachten und zu verstärken, die Schüler zu eigenständigem, selbstverantwortlichem Lernen und Arbeiten anzuleiten und Teamfähigkeit, auch in klassen- und jahrgangsstufenübergreifenden Projekten, zu fördern. 
Die Schüler müssen im Gegenzug die grundlegende Bereitschaft aufbringen, sich auf neue Inhalte interessiert einzulassen, zunehmend den Unterricht mitzugestalten, sich selbst aktiv in das Schulleben einzubringen und aufgeschlossen und unvoreingenommen auf ihre Mitschüler zuzugehen.

Auf dieser Basis werden die Kinder und Jugendlichen dabei unterstützt, ihre Stärken zu entwickeln, aber auch ihre Schwächen selbstkritisch wahrzunehmen und zu verringern.

Wichtig sind dabei auch ein respektvoller Umgang miteinander sowie die Einhaltung gepflegter Umgangsformen. Im täglichen Miteinander müssen persönliche Grenzen aufgezeigt und muss für deren Einhaltung gesorgt werden. Kritik muss fair und sachgerecht als wichtige Rückmeldung zu Inhalten oder Verhalten geäußert werden und darf keinesfalls zur Abwertung der Person führen. Die Erwartung geht dahin, dass jeder sein Handeln reflektiert und bereit ist, auch von anderen zu lernen.

Dazu ist es zwingend notwendig, Personen in ihrer Gesamtheit wertschätzend wahrzunehmen und zu achten.

Dieses bedeutet im Einzelnen, Individualität zu unterstützen, indem Talente erkannt und eigene Ideen gefördert sowie Erfahrungen miteinander reflektiert werden. Zudem müssen den Schülern Vorbilder angeboten werden, die eigene Werte leben, als Menschen sichtbar werden und als Gesprächspartner gerade auch in schwierigen Situationen helfen. Dies funktioniert nur unter Herstellung guter Rahmenbedingungen, indem grundsätzlich eine positive Einstellung zueinander gepflegt und problemlösungsorientiert gehandelt wird.

Ein lebendiges Miteinander zwischen Schülern, Lehrern, Assistenten, Eltern, Außenwohngruppen und Therapeuten gelingt, indem Spontaneität und Kreativität zugelassen und gelebt werden.

Grundlage dafür ist wiederum eine hohe Qualität der Arbeit, insbesondere Fachwissen und regelmäßige Fortbildungen in allen Bereichen pädagogischen Handelns. Wichtig sind auch der ständige Kontakt in Bayern zum Staatsministerium für Unterricht und Kultus und zu den Bezirksregierungen, der Kontakt zu anderen Schulen, insbesondere zur Grund-/Haupt-und Fachoberschule im eigenen Haus, die Umsetzung des Lehrplans für die sechsstufige Realschule in Bayern, eine kontinuierliche Schulentwicklung sowie gegenseitige kollegiale Unterstützung bei der Umsetzung aller Ziele und Teamfähigkeit.

Es muss ferner eine Zusammenarbeit mit den Schülern, Assistenten-Teams, Schulbegleitern, Erziehungsberechtigten, den Betreuern in Stationären Wohngruppen und Heilpädagogischen Tagesstätten, Ärztlichem Dienst, Physio-und Ergotherapie, Logopädie, Psychologischem Dienst, Sozial-und Beratungsdienst und schließlich externen Beratungsdiensten, wie Mobilen Sozialen Diensten, Staatlicher Schulberatung, der Bundesagentur für Arbeit und den Jugendämtern stattfinden.

Gute Arbeit gelingt nur durch eine konsequente Überprüfung und Weiterentwicklung vorhandener Kommunikationsstrukturen und Arbeitsabläufe. 
Bauliche, technische und organisatorische Voraussetzungen dafür sind barrierefreie Schulgebäude, behindertengerechte Schwimmbäder und Sporthallen, Räumlichkeiten zur Differenzierung, Schulküchen mit unterfahrbarer und höhenverstellbarer Einrichtung, verstellbare Tische und Spezialstühle, die Verwendung von technischen Hilfsmitteln, wie etwa Notebooks, Beamer, Overheadprojektoren, Lesegeräte, Kommunikatoren, im Unterricht und bei Prüfungen, ein Mittagsrestaurant, Stadtteilbibliotheken in den Schulgebäuden sowie Fahrdienste für behinderte Schüler.

Pädagogische Rahmenbedingungen dafür sind geringe Klassenstärken (ca. 15 Schüler), die Integration auch nichtbehinderter Schüler, ein Unterricht im Fachlehrersystem mit einer Unterrichtsbegleitung durch ein eigenes Assistenten-Team, welches bei Bedarf Schüler unterstützt, Nachteilsausgleiche, individuelle behinderungsbedingte Sonderregelungen, z.B. verkürzte Unterrichtszeit, Heimunterricht, Therapie statt Unterricht, die Stärkung der eigenständigen Arbeitsweise der Schüler durch unterstützende Unterrichtsformen und nicht zuletzt eine starke Binnendifferenzierung im Unterricht. 
